\title{
Introduction to the Learning in Digital and Social Media Minitrack
}

\author{
Maarten de Laat \\ University of Wollongong \\ mdelaat@uow.edu.au
}

\author{
Dan Suthers \\ University of Hawaii \\ suthers@hawaii.edu
}

Learning in Digital and Social Media is an interdisciplinary minitrack to present research on how human learning takes place via interactive and social processes enabled or supported by digital and social media. We seek to bridge disciplines and research communities between system and learning sciences and within this scope a broad range of research questions, learning settings, and theoretical and methodological traditions are welcome. The shared focus is on relationships between human learning activities and the technologies used: the designed or discovered affordances of digital media that can be taken up to support learning through interaction with others.

This year the Learning in Digital and Social Media minitrack consists of three highly valued papers. Each of these papers successfully address complex methodological challenges of researching online learning through social interaction in digital media and present innovative designs for social learning. The first paper, presented by Amelia Acker and Leanne Bowler with the title "Youth Data Literacy: Teen Perspectives on Data Created with Social Media and Mobile Devices", explores how teenagers conceptualize data. Their focus is on what it means to grow up in a world where owning mobile devices and building multiple data profiles is part of everyday life. They are interested in how teenagers learn about data through the use of social media platforms, creation of mobile media, and ownership of mobile devices. In their ethnographic study they draw on data from participants (11-18 years old) using public spaces such as libraries to work on projects, hang out and learn. The paper presents findings about how teenagers learn and acquire knowledge about the interactive and social processes of the data lifecycle in public spaces and social media, with a particular interest on their learning about data awareness through sharing, aging and owning mobile devices. This research contributes to our theoretical understanding of data management and people's learning to make sense of data and how it influences their lives. The notion of participation in public spaces and how it influences and changes our ability to learn is further explored in the next paper. "Learning in the Wild: Coding Reddit for Learning and Practice", our best paper nomination, presented by Priya Kumar, Anatoliy Gruz, Caroline Haythornthwaite, Sara Gilbert, Marc Esteve del Valle and Drew Paulin, introduces a coding schema developed to support learning researchers' ability to conduct research into learning in social and digital media. Their particular interest is in informal learning environments and the coding schema aims to provide insight in how knowledge, ideas and resources are being shared and supported outside the confines of traditional education and professional development. Based on the exploratory talk framework they started with human coding of content in Reddit communities in order to prepare the coding schema for automated analysis. This paper discusses how the coding schema was developed and presents an innovative way into understanding social learning through dialogues in digital media. The researchers provide clear insight into the coding schema and how it can be applied by other scholars to collectively advance our knowledge in this domain. The third and final paper of this session is about "Peer and Vicarious Framing, Problematization, and Situated Learning in Online Professional Master Courses" and is presented by Susan Gasson and Jonan Donaldson. This paper explores modes of social interaction and conditions for vicarious learning in an online professionally-oriented project management course aimed at engaging students in situated learning processes that reflect social identity, cultural values and practices of professional communities. Based on coding of community content and social network analysis they identified that processes of knowledge building and framing of collective knowledge depends on experienced community participants that act as thought-leaders and can contribute vicarious knowledge to the joint community process of sensemaking. 